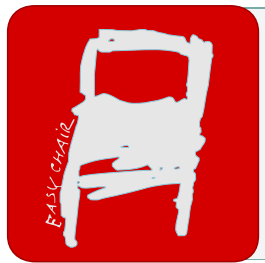

EPiC Series in Health Sciences

Volume 4, 2020, Pages 293-296

CAOS 2020. The 20th Annual Meeting of the International Society for Computer Assisted Orthopaedic Surgery

\title{
The Effect of Coronal and Axial Femoral Component Rotation on Midflexion Laxity and Patient Reported Outcomes in Total Knee Arthroplasty
}

\author{
Edgar A Wakelin ${ }^{1}$, Sami Shalhoub ${ }^{1}$, Jeffrey M. Lawrence ${ }^{2}$, John M. Keggi ${ }^{3}$, \\ Amber L. Randall ${ }^{4}$, Corey E. Ponder ${ }^{5}$, Jeffrey H. DeClaire ${ }^{6}$, Jan Koenig ${ }^{7}$, \\ Christopher Plaskos ${ }^{1}$ \\ ${ }^{1}$ Corin, Raynham, MA \\ ${ }^{2}$ Gundersen Health System, Viroqua, WI \\ ${ }^{3}$ Orthopaedics New England, Middlebury, CT \\ ${ }^{4}$ Flagstaff Bone and Joint, Flagstaff, AZ \\ ${ }^{5}$ Oklahoma Sports and Orthopedics Institute, Oklahoma, OK \\ ${ }^{6}$ DeClaire LaMacchia Orthopaedic Institute, Rochester Hills, MI \\ ${ }^{7}$ NYU-Winthrop, Mineola, NY \\ edgar.wakelin@coringroup.com
}

\begin{abstract}
Joint balance in total knee arthroplasty (TKA) has traditionally focused on achieving a tight symmetric extension gap and rectangular or trapezoidal gaps in flexion. This study sought to investigate the effect of femoral and tibial coronal rotation and femoral axial rotation on midflexion coronal joint balance and patient outcomes.

A prospective multi-center study was performed with a mixture of tibia-first gapbalancing and femur-first approaches were performed using the Corin OMNIBotics robot-assisted TKA platform with APEX implant components. Coronal and axial femoral and tibial resections were recorded by the platform. Medial and lateral joint gaps were recorded while applying a computer-controlled load to the joint throughout flexion during trialing using the Corin BalanceBot device. In addition, 1-year Knee Injury and Osteoarthritis Outcome Score (KOOS) and PROMIS-10 global health scores were collected.

231 surgeries were identified: $66.9 \pm 8.1$ years, $31.4 \pm 4.8 \mathrm{~kg} / \mathrm{m} 2$ and $57 \%$ female (121) with a mean pre-operative HKA angle of $4.5 \pm 5.2^{\circ}$ varus. A significant correlation was found between the medio-lateral (ML) joint gap difference in midflexion and both extension and flexion joint line ( $\mathrm{p}=0.003, \mathrm{r} 2=-0.20, \mathrm{p}=0.001, \mathrm{r} 2=-0.22$, respectively). A significant correlation was found between midflexion ML imbalance and KOOS stiffness
\end{abstract}


The Effect of Coronal and Axial Femoral Component Rotation on Midflexion Laxity... Wakelin et al.

questions at $3 \mathrm{M}$ and $6 \mathrm{M}$ post-op $(\mathrm{r} 2=-0.15, \mathrm{p}=0.036, \mathrm{r} 2=-0.18, \mathrm{p}=0.013)$, in which a more balanced knee correlated with improved outcomes.

Treating flexion and extension joint balance in isolation may not capture the effect on midflexion laxity. Component placement should take in to account the effect on joint gaps throughout flexion to target optimal joint balance.

\section{INTRODUCTION:}

Joint balance in total knee arthroplasty (TKA) has traditionally focused on achieving a tight symmetric extension gap and either rectangular or trapezoidal gaps in flexion. Joint gap targets however have also been affected by an historic lack of technology available to accurately quantify joint balance at a range of flexion angles [1]. Modification to midflexion balance resulting from TKA has been particularly difficult to quantify intra-operatively due to the complex nature of the relationship between three-dimensional sagittal and coronal rotation of the femoral and tibial components and patient specific soft tissue profile [2]. This study sought to investigate the effect of femoral and tibial coronal rotation and femoral axial rotation on midflexion coronal joint balance and patient outcomes.

\section{METHODS:}

A prospective multi-center study was performed with a mixture of tibia first gap balancing and femur first (targeting mechanical alignment) approaches were performed using the Corin OMNIBotics robot (Corin USA, Raynham, MA) assisted TKA platform with APEX implant components. Coronal and axial femoral and tibial resections were recorded by the platform. Medial and lateral joint gaps were recorded while applying a computer-controlled load to the joint throughout the range of motion during trialing using the Corin BalanceBot device (Corin USA, Raynham, MA). To control for the effect of tibial coronal resection on midflexion laxity, the joint line was calculated as the average between the tibial and femoral coronal resection for the extension joint line, and the average between the tibial coronal resection and femoral axial resection for the flexion joint line, similar to that described by Bellemans et. al [3]. In addition, 1-year Knee Injury and Osteoarthritis Outcome Score (KOOS) scores were collected.

Correlations between the validated resection angle recorded by the OMNIBotics platform, joint gaps in midflexion $\left(30^{\circ}-50^{\circ}\right)$ and KOOS scores were calculated using Spearman's correlation coefficients, and Wilcox rank-sum tests were performed to determine the difference between categorized gap data. All statistical analysis was performed using R 3.5.3 (R Core Team, Vienna, Austria).

\section{RESULTS:}

231 surgeries were identified with all data. The population was $66.9 \pm 8.1$ years, BMI: $31.4 \pm 4.8 \mathrm{~kg} / \mathrm{m}^{2}$ and $57 \%$ female (121) with a mean pre-operative HKA angle of $4.5 \pm 5.2^{\circ}$ varus. Tibial coronal resection of $0.36 \pm 0.90^{\circ}$ varus combined with a femoral coronal resection of $0.6 \pm 1.99^{\circ}$ varus and femoral axial resection of $2.14 \pm 2.63^{\circ}$ external rotation from the posterior condyles, resulted in a postop extension coronal angle of $0.97 \pm 2.23^{\circ}$ varus and flexion angle of $2.50 \pm 2.70^{\circ}$ varus. A significant correlation was found between the medio-lateral (ML) joint gap difference in midflexion and extension joint line $\left(\mathrm{p}=0.003, \mathrm{r}^{2}=-0.20\right)$, in which a more medially down sloped joint line (corresponding to a tibial varus and femoral valgus resection) resulted in a greater lateral opening of the joint. Similarly, a significant correlation was found for the flexion joint line $\left(p=0.001, r^{2}=-0.22\right)$ 
The Effect of Coronal and Axial Femoral Component Rotation on Midflexion Laxity... Wakelin et al.

in which a more medially down sloped joint line (corresponding to a tibial varus and femoral internal rotation resection) resulted in greater lateral opening in midflexion.

A significant correlation was found between midflexion ML imbalance and KOOS stiffness questions (S6 \& S7) at 3 Months and 6 Months post-op $\left(r^{2}=-0.15, p=0.036, r^{2}=-0.18, p=0.013\right)$, in which a more balanced knee correlated with improved outcomes. Patients with a gap imbalance of less than $1.5 \mathrm{~mm}$, reported significantly better stiffness outcomes $(74.9 \mathrm{vs} 68.9, \mathrm{p}=0.038)$ at 6 Months, Figure 1.

\section{DISCUSSION:}

This study found significant correlations between the flexion and extension joint line and midflexion gaps. Furthermore, the midflexion gap was found to significantly correlate with patient reported stiffness outcomes at $3 \mathrm{M}$ and $6 \mathrm{M}$ post-op. This data suggests that flexion and extension laxity should not be considered in isolation during TKA and should incorporate the effect on midflexion laxity when planning component placement. Midflexion balance has previously been reported as independent of both flexion and extension laxity due to the contributions of the anterior and posterior soft tissues [4], and difficult to measure without digital tools. Improvement in intra-operative technology now allows the three-dimensional effect of component placement on joint gaps throughout flexion to be accurately measured in real time [5].

There are limitations to this study, for example, the effect of pre-operative anatomy (e.g. bony morphology) on component placement and resulting joint laxity has not been investigated here. More highly deformed knees may benefit from a different component alignment or soft tissue target balance [6]. The effect of anatomy on ideal component placement is the subject of ongoing analysis. Furthermore, the joint distraction force for measuring gaps was between $70-90 \mathrm{~N}$, it is unknown if this force is ideal, or should be patient specific.

Treating flexion and extension joint balance in isolation may not capture the effect on midflexion laxity. Component placement should take in to account the effect on joint gaps throughout flexion to target optimal joint balance.

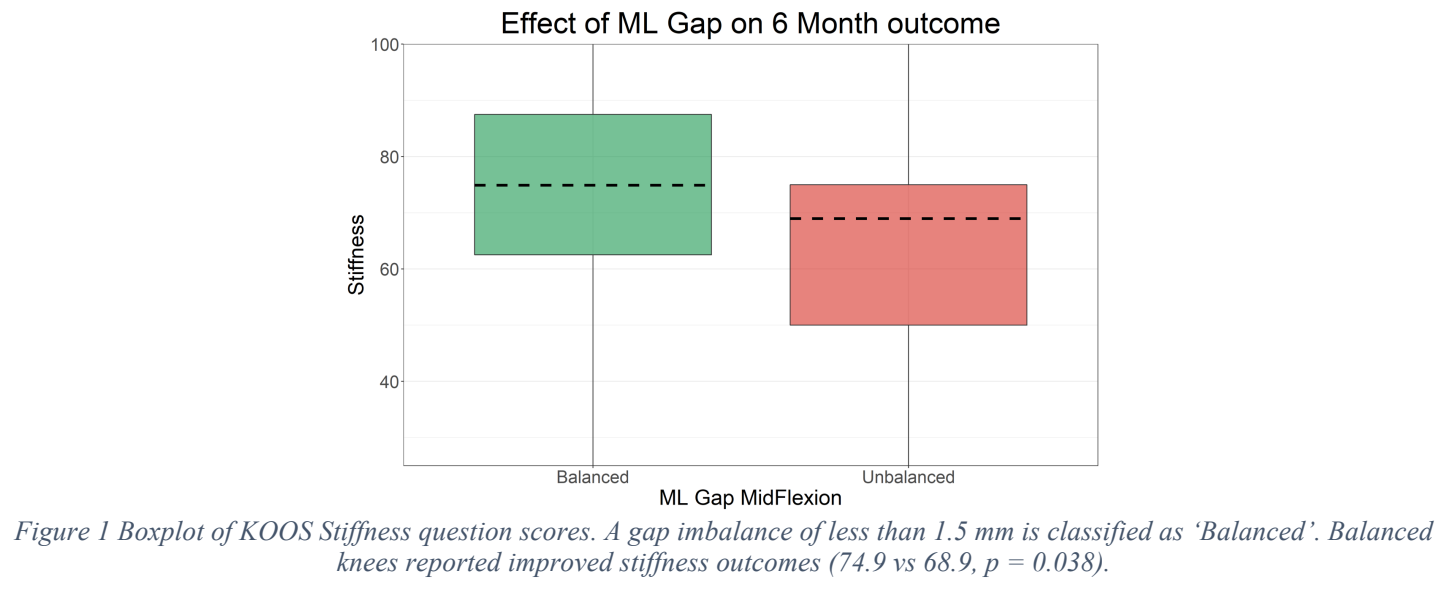


The Effect of Coronal and Axial Femoral Component Rotation on Midflexion Laxity... Wakelin et al.

\section{REFERENCES:}

1. Roth, J.D. and S.M. Howell, Soft tissue balance of the native knee provides guidance for balancing a total knee arthroplasty, in Soft Tissue Balancing in Total Knee Arthroplasty. 2017, Springer. p. 17-27.

2. Nagle, M. and A. Glynn, Midflexion Instability in Primary Total Knee Arthroplasty: A Review Article. The journal of knee surgery, 2019.

3. Victor, J.M., et al., Constitutional varus does not affect joint line orientation in the coronal plane. Clinical Orthopaedics and Related Research®, 2014. 472(1): p. 98-104.

4. Vince, K., Mid-flexion instability after total knee arthroplasty: woolly thinking or a real concern? The bone \& joint journal, 2016. 98(1_Supple_A): p. 84-88.

5. Shalhoub, S., et al., Imageless, robotic-assisted total knee arthroplasty combined with a robotic tensioning system can help predict and achieve accurate postoperative ligament balance. Arthroplasty Today, 2019.

6. Nishida, K., et al., Remaining mild varus limb alignment leads to better clinical outcome in total knee arthroplasty for varus osteoarthritis. Knee Surgery, Sports Traumatology, Arthroscopy, 2017. 25(11): p. 3488-3494. 